\title{
Simulación numérica de ensayos de compresión diametral para la evaluación de discos cerámicos porosos
}

\author{
M. L. SANDOVAL, A. G. TOMBA MARTINEZ, M. A. CAMERUCCI
}

Laboratorio de Materiales Estructurales - División Cerámicos, INTEMA-CONICET

Facultad de Ingeniería-UNMdP. Av. Juan B. Justo 4302 (7600) Mar del Plata, Argentina.

\begin{abstract}
Se estudió el comportamiento mecánico de materiales porosos de cordierita mediante ensayos en compresión diametral. La solución analítica que permite la medición indirecta de la resistencia mecánica a tracción en compresión diametral se formula bajo ciertas condiciones que no suelen satisfacerse en la práctica. Con el objetivo de analizar desviaciones de las condiciones ideales se simuló el ensayo con técnicas computacionales. Los discos porosos de cordierita se obtuvieron por calcinación $\left(650{ }^{\circ} \mathrm{C}, 2 \mathrm{~h}\right)$ y reacción-sinterización $\left(1330^{\circ} \mathrm{C}, 4 \mathrm{~h}\right)$ de cuerpos en verde preparados por consolidación térmica $\left(85^{\circ} \mathrm{C}, 4 \mathrm{~h}\right)$ de suspensiones acuosas de una mezcla precursora de cordierita (caolín, talco y alúmina) con almidón nativo de papa. Los ensayos mecánicos se realizaron en una máquina servohidráulica en control por desplazamiento $(0,2 \mathrm{~mm} / \mathrm{min})$. A partir de la relación aparente tensión-deformación se determinaron los parámetros mecánicos: módulo de Young aparente, resistencia mecánica y límite elástico. Sobre los discos ensayados se analizaron las características de la fractura. La influencia de las desviaciones geométricas más frecuentes identificadas en la práctica (desviación de la circularidad y no paralelismo entre las caras del disco) sobre la distribución de tensiones se estudió mediante la simulación por elementos finitos, tomando como referencia la ecuación de Hertz para problemas de contacto.
\end{abstract}

Palabras claves: Compresión diametral, Simulación numérica, Cerámicos porosos, Cordierita.

Numerical simulation of diametral compression tests for the evaluation of porous ceramic disks

The mechanical behavior of porous cordierite materials was studied by diametral compression tests. The analytical solution allowing the indirect measuring of the tensile mechanical strength in this load configuration is formulated under certain assumption which may be not satisfied in practice. With the aim to analyze deviations of the ideal conditions, the test was simulated using computational techniques. Porous cordierite disks were prepared by firing $\left(650{ }^{\circ} \mathrm{C}, 2 \mathrm{~h}\right)$ and reaction-sintering $\left(1330^{\circ} \mathrm{C}, 4 \mathrm{~h}\right)$ of green disks shaped by thermogelling the aqueous suspensions of a cordierite precursor mixture (kaolin, talc and alumina) with native potato starch as a consolidator/binder of ceramic particles and a pore former by burn-out at high temperature. The mechanical tests were carried out in displacement control $(0.2 \mathrm{~mm} / \mathrm{min})$ using a servohydraulic testing machine. From the apparent stress-strain ratio, the following parameters were determined: mechanical strength, apparent Young modulus and yield stress. Fracture features of tested disks were also analyzed. The influence of the geometrical deviations more usually identified in practice (deviation of the circularity and no parallelism between the plane surfaces of the disk) on the stress distribution was studied by means of the simulation by finite element method, considering the Hertz's equation for contact problems as reference.

Keywords: Diametral compression, Numerical simulation, Porous ceramics, Cordierite.

\section{INTRODUCCIÓN}

La alta demanda de materiales cerámicos porosos con porosidad controlada que reúnan propiedades específicas (mecánicas y térmicas, entre las más relevantes) para determinadas aplicaciones ha impulsado el desarrollo de nuevos métodos de procesamiento $(1,2)$. En particular, el estudio del rendimiento de estos materiales en aplicaciones estructurales debe incluir necesariamente la evaluación de su comportamiento mecánico en relación a ciertas características microestructurales como la cantidad, la morfología y la distribución de tamaños de poros. Estas características son las que generalmente causan un efecto determinante sobre las propiedades finales del material, en particular las mecánicas (3). En la evaluación experimental del comportamiento mecánico de estos materiales, resulta indispensable contar con ensayos que provean datos confiables y reproducibles.

Una de las configuraciones usadas para la evaluación mecánica de cerámicos en verde y sinterizados, es la compresión diametral de discos (4-12), también denominado 'splitting test' o 'Brazilian test', debido a sus varias ventajas frente a técnicas similares (flexión en 3 y 4 puntos): simplicidad en la configuración de carga y geometría de probeta, ausencia de efectos de borde, independencia del acabado superficial, entre las más importantes. Este 
ensayo mecánico consiste en la aplicación de una carga compresiva uniaxial sobre una probeta de geometría cilíndrica, distribuida a lo largo de dos generatrices diametralmente opuestas, hasta alcanzar la rotura de la probeta. Las expresiones matemáticas para describir el estado bidimensional de tensiones generado en la probeta fueron desarrolladas por Hertz bajo condiciones de carga puntual $(5,6,9,11)$. Según su teoría, la falla se producirá a través de una fisura central iniciada en el centro de la probeta (punto del máximo esfuerzo principal en tracción) que propagará diametralmente hacia la periferia del disco. En la realidad, debido a las altas tensiones compresivas y de corte que se generan en la zona cargada, existe la posibilidad de que la fractura se inicie en estos puntos, produciendo aplastamiento ('crushing') del material o delaminación, respectivamente. Por su parte, la deformación elástica o plástica local en la zona de contacto con los platos de compresión distribuye la carga sobre una cierta área de tamaño finito lo que afecta el campo de tensiones.

Basado en las mismas suposiciones que asume Hertz respecto al material, el contacto y el estado de tensiones, Hondros introduce la condición de carga distribuida (6, 9). Matemáticamente, ambos análisis son exactos para el problema elástico y satisfacen las ecuaciones de equilibrio. Si bien cerca del centro, la distribución de tensiones en los modelos de Hertz y Hondros son muy similares, cerca de los puntos de aplicación de la carga, la tensión horizontal (perpendicular a la dirección de aplicación de la carga) se hace compresiva, mientras que los valores verticales son considerablemente menores que los calculados para la condición de carga puntual. La solución propuesta por Hondros muestra que la distribución de la carga afectará el valor de tensión máximo en tracción respecto a la condición de carga puntual: la distribución de la carga sobre un arco con apertura angular mayor a $30^{\circ}$ produce una desviación de la solución mayor al 10\% respecto de considerar carga puntual.

Las soluciones analíticas del estado de tensiones desarrollado en el interior de una probeta sometida a compresión diametral, y que permiten la medición indirecta de las propiedades mecánicas, están fundadas sobre varias hipótesis que no siempre se satisfacen en la práctica: geometría cilíndrica regular, comportamiento elástico-lineal y contacto sin fricción, entre otras. Las desviaciones de estas condiciones pueden invalidar el ensayo para la obtención de la resistencia mecánica a tracción, con lo cual se requiere llevar a cabo un análisis exhaustivo del mismo. El empleo de técnicas computacionales para analizar la influencia del alejamiento del caso ideal sobre los resultados del ensayo mecánico resulta una herramienta muy potente $(9,13)$.

Las desviaciones con respecto a la geometría cilíndrica de la probeta ensayada, algunas de las cuales están en parte relacionadas con el procesamiento empleado y las características del material cerámico, inciden en la determinación de los parámetros mecánicos mediante el ensayo de compresión, así como otros factores experimentales como las características de la zona de contacto (uso y características del material 'pad', lo cual define el área de contacto, y agentes lubricantes), la velocidad de aplicación de la carga, la relación diámetro/espesor de la probeta, entre otros. En la evaluación mecánica de discos porosos de cordierita obtenidos por consolidación térmica de suspensiones acuosas de una mezcla precursora con almidón y posterior reacción-sinterización, realizada bajo las mismas condiciones experimentales (velocidad de aplicación de la carga, relación diámetro/espesor, agente lubricante y material 'pad'), se detectaron como desviaciones geométricas más frecuentes la falta de circularidad del perímetro del disco y de paralelismo entre las superficies planas del mismo. El objetivo de este trabajo es analizar la influencia de estas irregularidades sobre la distribución de las tensiones generadas mediante la simulación numérica del ensayo por el método de elementos finitos (MEF), tomando como referencia la solución de Hertz.

\section{PARTE EXPERIMENTAL}

\subsection{Materiales}

Se prepararon discos en verde por gelificación térmica $\left(85{ }^{\circ} \mathrm{C}, 4 \mathrm{~h}\right)$ de una suspensión acuosa $(29,6 \%$ vol.) de polvos comerciales de caolín, talco micronizado y alúmina (mezcla precursora de cordierita) con almidón comercial de papa (11,5\% vol.) y secado a $50{ }^{\circ} \mathrm{C}, 12 \mathrm{~h}$, empleando moldes impermeables de acero inoxidable (14-16). En el procesamiento empleado el almidón actúa como agente consolidante/ligante de la suspensión cerámica y formador de poros a alta temperatura. La mezcla precursora se formuló en una proporción (37\% de caolín, $41 \%$ de talco y $22 \%$ de alúmina, en peso) cercana a la de la cordierita estequiométrica $\left(51,4 \%\right.$ de $\mathrm{SiO}_{2}, 34,8 \%$ de $\mathrm{Al}_{2} \mathrm{O}_{3}$ y $13,8 \%$ de $\mathrm{MgO}$, en peso).

Los discos en verde, una vez mecanizados con broca de acero, se trataron térmicamente en aire (horno eléctrico con elementos calefactores de $\mathrm{SiC}$, Carbolite) empleando el siguiente ciclo: $1{ }^{\circ} \mathrm{C} / \mathrm{min}$ hasta $650^{\circ} \mathrm{C}, 2 \mathrm{~h} ; 3^{\circ} \mathrm{C} / \mathrm{min}$ hasta $1330{ }^{\circ} \mathrm{C}$, 4 h y enfriamiento a $5{ }^{\circ} \mathrm{C} / \mathrm{min}$ hasta temperatura ambiente. Así, se obtuvieron discos basados en cordierita de 2-4 mm de altura y 12-16 mm de diámetro con 1,17 $\pm 0,04 \mathrm{~g} /$ $\mathrm{cm}^{3}$ de densidad aparente y $57 \pm 2 \%$ de porosidad.

\subsection{Ensayo de compresión diametral}

Los ensayos en compresión diametral se realizaron en una máquina servohidráulica INSTRON modelo 8501, con celda de carga de $5 \mathrm{kN}$ y platos de compresión de acero (HRC 65) (14). Para reducir los efectos de fricción y concentración de tensiones en estos puntos, se colocó grasa de $\mathrm{MoS}_{2}$ entre la probeta y los platos de compresión. Además, entre el disco y los platos se colocó un papel blanco y un papel carbónico para producir una distribución adecuada de la carga (material 'pad') (5) y evaluar simultáneamente el ancho $(w)$ en el que se distribuye la carga. Los ensayos se realizaron en aire a temperatura ambiente, en control por desplazamiento, con una velocidad de 0,2 $\mathrm{mm} / \mathrm{min}$. En estas condiciones se ensayaron ocho probetas, considerado un número estadístico de muestras.

A partir de los ensayos se obtuvieron las curvas cargadesplazamiento y a partir de las mismas se calculó la relación aparente tensión $(\sigma)$-deformación $(\varepsilon)$. Los valores de tensión se calcularon mediante la ecuación (1) (4-6): 


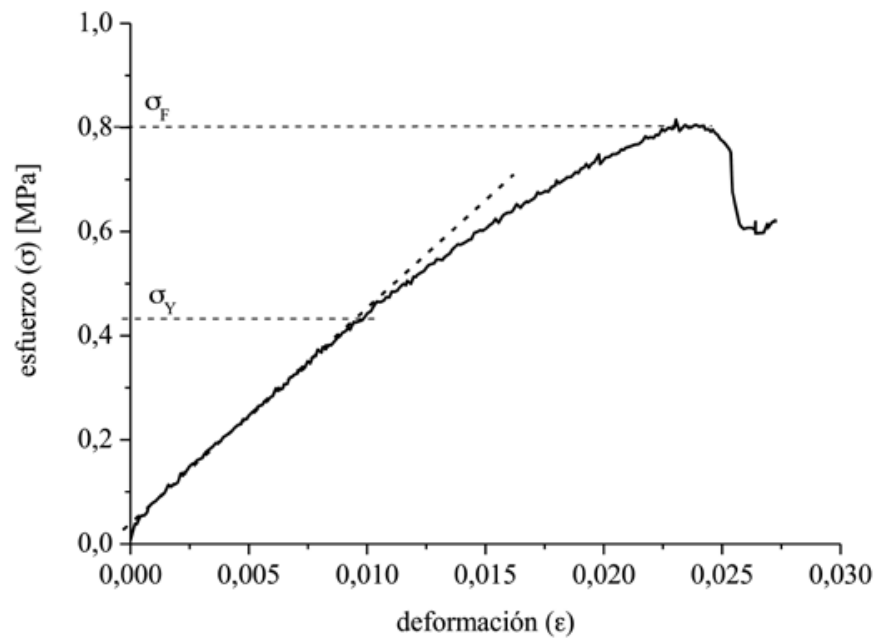

Figura 1. Curva tensión-deformación típica para un disco poroso de cordierita.

$$
\sigma=\frac{2 P}{\pi D L}
$$

donde $P$ es la carga aplicada y $D$ y $L$ son el diámetro y el ancho del disco, respectivamente. La deformación se consideró como el cociente entre el desplazamiento del actuador y el diámetro del disco. De las curvas $\sigma-\varepsilon$; de las cuales se muestra un ejemplo típico en la Figura 1, se determinaron el módulo de Young aparente $\left(\mathrm{E}_{\mathrm{a}}\right)$ como la pendiente de la parte lineal de la curva, la resistencia a la tracción $\left(\sigma_{\mathrm{F}}\right)$, definida como la tensión normal necesaria para que el material falle en el modo I y equivalente al valor máximo de $\sigma$, y el límite elástico aparente $\left(\sigma_{\gamma}\right)$ como el valor de la tensión que corresponde a un apartamiento en deformación del $1 \%$ respecto del comportamiento elástico lineal (Figura 1). La relación $\sigma_{\mathrm{Y}} / \sigma_{\mathrm{F}}$ se consideró un indicador del grado de desviación del comportamiento lineal por deformación permanente.

Los valores de los parámetros mecánicos extraídos de las curvas $\sigma-\varepsilon$ para los discos ensayados resultaron: $\sigma_{\mathrm{F}}=$ $10,6 \pm 1,5 \mathrm{MPa}, \mathrm{Ea}=580 \pm 160 \mathrm{MPa}$ y $\sigma_{\mathrm{Y}} / \sigma_{\mathrm{F}}=73 \pm 21 \%$.
Considerando la corrección por la porosidad al valor de la resistencia mecánica dado por la ecuación (2) (3):

$$
\sigma_{F}=\sigma_{F}^{0} \exp (-b p)
$$

donde $\sigma_{\mathrm{F}}^{0}$ es la resistencia a la fractura para un valor nulo de porosidad, $p$ es la fracción volumétrica de poros y $b$ es un coeficiente que depende del tamaño y morfología del poro, el valor de $\sigma$ resulta consistente con los datos de literatura para materiales de cordierita densos ensayados en flexión en 3 puntos (70-80 $\mathrm{MPa})(17)$, teniendo en cuenta que los valores de resistencia a la fractura determinados por compresión diametral son siempre menores a los determinados en flexión $(8,11)$. De manera similar, a partir de la consideración del efecto de la porosidad sobre el módulo elástico con una relación análoga a la ecuación (2) (3):

$$
E a=E a^{0} \exp (-b p)
$$

se obtuvo un valor de $\mathrm{Ea}^{0}$ muy cercano a valores reportados en la literatura para materiales basados en cordierita (135$140 \mathrm{GPa}$ ) (18). Respecto a la relación $\sigma_{\mathrm{Y}} / \sigma_{\mathrm{F}}$, su valor manifiesta un comportamiento esencialmente frágil, con alguna incidencia de los procesos de microfisuración o desgranamiento del material en la zona de contacto, que lleva a la desviación de la linealidad observada en las curvas tensión-deformación (Figura 1).

El tratamiento teórico del que surge la ecuación (1) considera, entre otras cosas, la condición de carga puntual $(5,6,9,11)$. Sin embargo, en la práctica la carga se distribuye sobre un área finita de ancho $w$ modificando la distribución de tensiones en el disco (6) en una magnitud que depende, aunque no exclusivamente, del valor de $w / D$. Así, la validez de la ecuación (1) empleada para calcular la resistencia mecánica depende del valor de esta relación y no hay un criterio consensuado sobre el rango de valores de $w / D$ que deben ser usados para validar el cálculo. Para el caso de los discos ensayados, se estimó un valor de $w / D$ de $0,03 \pm 0,01$, con lo cual el error en el cálculo de la resistencia mecánica originado en la distribución de la carga resultó menor al 1,5\% $(6,10)$.

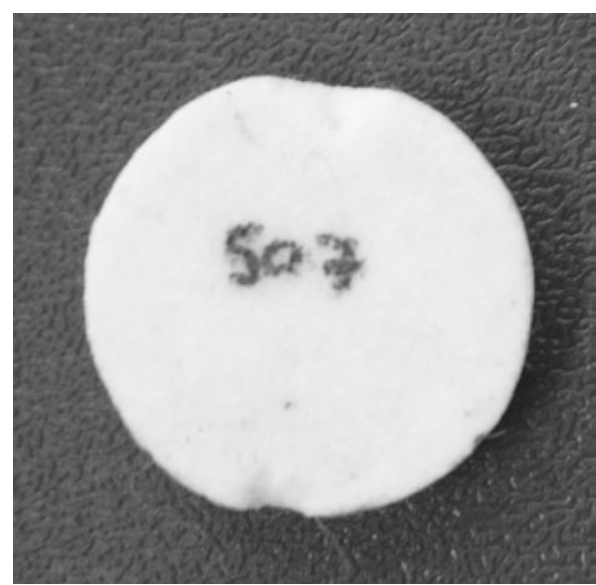

a)

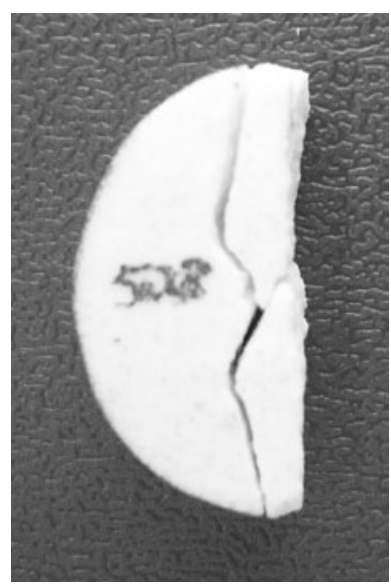

b)

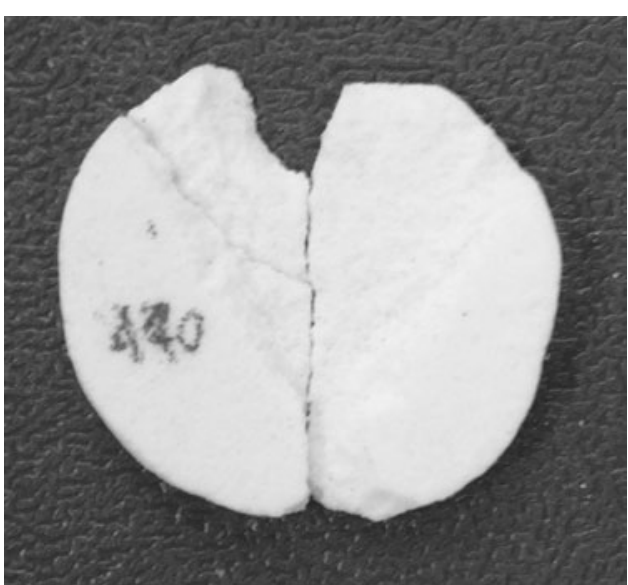

c)

Figura 2. Patrones de fractura típicos: a) delaminación en la zona de contacto; b) ‘triple-cleft'; c) fractura diametral más delaminación en zona de contacto. 
Luego de los ensayos, se determinó el patrón de fractura característico y se realizó el análisis fractográfico por microscopía electrónica de barrido (MEB, Jeol JSM-6460, Japan). Los discos ensayados presentaron los siguientes patrones de fractura (entre paréntesis se informa la frecuencia de aparición), que se muestran en la Figura 2: delaminación en la región de contacto $(50 \%)$, 'triple-cleft' $(4,5)(25 \%)$ y fractura diametral junto con delaminación en la región de contacto $(25 \%)$. Si bien la validez de los ensayos en el caso de que la fractura no sea exclusivamente diametral es discutida por algunos autores (5), la rotura en 'triple-cleft' es comúnmente aceptada como un patrón válido $(4,12)$. En este caso, dado que las probetas que sólo sufrieron delaminación en la zona de contacto (sin fisura diametral) presentaron curvas $\sigma-\varepsilon$ con características similares a aquéllas correspondientes a los discos que fracturaron diametralmente (incluyendo el 'triple-cleft' como patrón de fractura válido), y propiedades mecánicas en el rango de valores de éstos últimos, no se las descartó para el cálculo de los valores medios de los parámetros mecánicos $\left(\sigma_{\mathrm{F}}\right.$, Ea y $\left.\sigma_{\gamma}\right)$.

En el análisis de las superficies de fractura en ningún caso fue posible determinar el defecto que originó la falla debido a la heterogeneidad microestructural de todas las superficies observadas y a la distribución de tensiones máximos de manera uniforme en el espesor como en el plano de carga (deformación plana). Aún así, se considera que el defecto limitante de la resistencia mecánica está asociado a la porosidad presente.

\subsection{Condiciones para el cálculo numérico}

Se analizaron los efectos que tiene la presencia de defectos geométricos en las probetas ensayadas sobre la distribución de tensiones generada durante el ensayo de compresión diametral. Específicamente, se estudiaron la falta de circularidad y el no paralelismo de las caras planas del disco por ser las que ocurrieron con mayor frecuencia en la práctica. A través de la implementación del método de elementos finitos, se simularon probetas de geometría cilíndrica de $4 \mathrm{~mm}$ de ancho y $16 \mathrm{~mm}$ de diámetro, en contacto con los platos de compresión, considerados como superficies indeformables. Para el cálculo se empleó un software comercial (ABAQUS 6.7).

Si bien el material que se simula presenta una microestructura heterogénea (contiene al menos la matriz sólida y poros), la morfología de la matriz tiene una distribución espacial aleatoria, que hace posible tratar al sólido como un material continuo y utilizar para el cálculo valores de propiedades globales. Basado en esto, se consideró para el modelo numérico un material homogéneo e isotrópico, con comportamiento elástico lineal (despreciando, en primera instancia, la desviación registrada en la práctica) y las siguientes propiedades: módulo de Young, E=580 MPa y módulo de Poisson, v=0,3. El valor del módulo de elasticidad se fijó teniendo en cuenta los valores experimentales obtenidos para los materiales estudiados y el valor de $v$ seleccionado es típico de materiales cerámicos. Las condiciones de contorno del problema fueron impuestas por los platos de compresión tal como ocurre en el sistema real, estableciendo entre la probeta y los platos una condición de contacto con un coeficiente de fricción $\mu_{\text {estático }}=0,3$ (16).
La magnitud de la carga fue fijada al imponer a uno de los platos un desplazamiento de $0,7 \mathrm{~mm}$ en la dirección $\mathrm{Y}$, manteniendo el otro fijo en su posición; esta magnitud se definió de manera tal que las deformaciones involucradas permitan evaluar el problema haciendo uso de la teoría elástica.

Aunque las simulaciones pretenden analizar pequeñas desviaciones respecto de la configuración ideal, se emplearon distintos modelos dependiendo del tipo de defecto. En cada caso se pudieron encontrar planos de simetría en el campo de tensiones, lo que permitió optimizar el recurso computacional y mejorar la solución.

La falta de circularidad se asimiló a un ovalamiento del disco. Este defecto se definió a través del parámetro diámetro menor/diámetro mayor, al cual se asignaron tres valores: 0,$90 ; 0,93 ; 0,96$. Además, se analizaron las distribuciones de tensiones considerando las distintas posiciones del eje mayor respecto del eje de aplicación de la carga, definidas por el ángulo $\theta$. Se empleó un modelo bidimensional considerando un estado de tensión plano y elementos de discretización triangulares de tres nodos, por su capacidad superior sobre los cuadrados a la hora de mallar geometrías intrincadas. Para describir la dirección de la tensión principal de tracción máxima en las probetas ovaladas, se definió el ángulo $\lambda$ que forman estas direcciones con el eje X.

Por su parte, para evaluar el no paralelismo de las caras planas (Figura 3), la geometría del perímetro se consideró circular, con ambas caras inclinadas un ángulo $\beta$ respecto de un plano de referencia. Dicho plano es perpendicular a las generatrices del disco y pasa por su centro. Se tomó como sistema de referencia una terna de ejes coordenados ortogonales con origen en el centro de la probeta, considerando a los ejes $\mathrm{X}$ e $\mathrm{Y}$ contenidos en el plano de referencia y al eje $Y$ coincidente con el de aplicación de la carga. La simetría del campo de tensiones permitió reducir el problema al estudio de medio disco (Figura 3), utilizando un modelo tridimensional, formado por elementos tetraédricos de cuatro nodos. Además de las

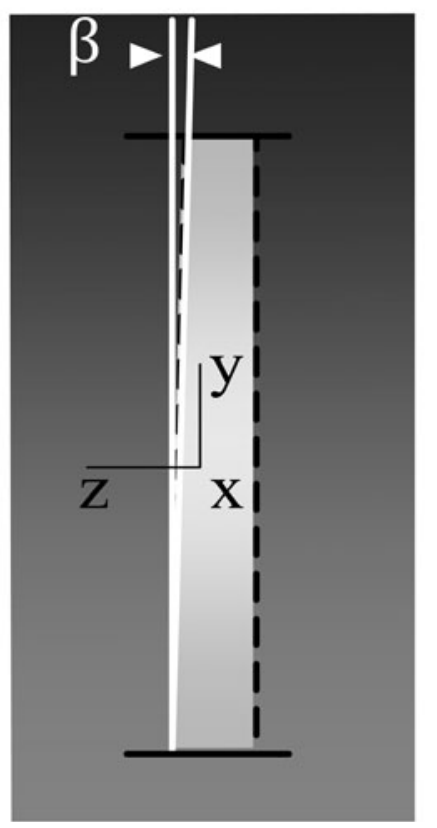

Figura 3. Modelo con caras no paralelas. 


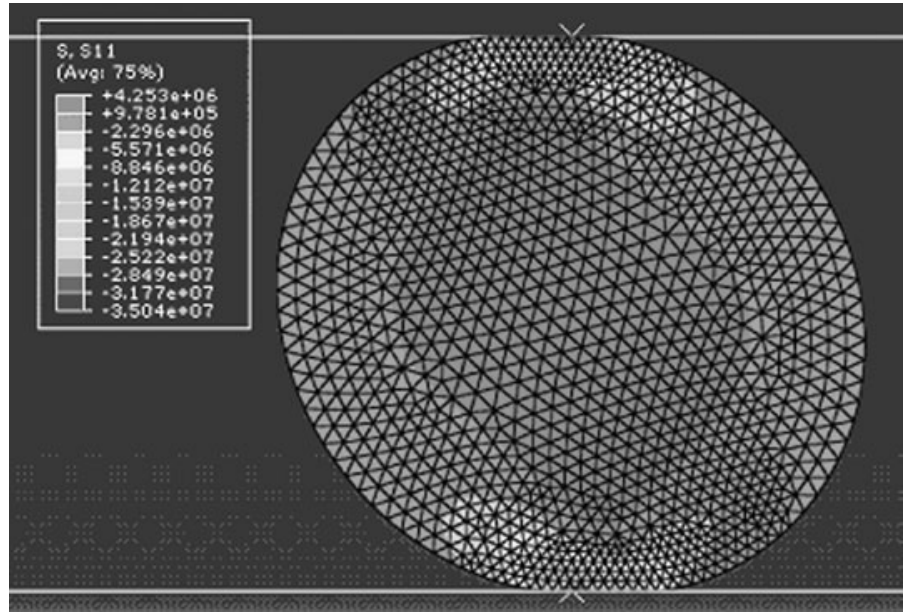

a)

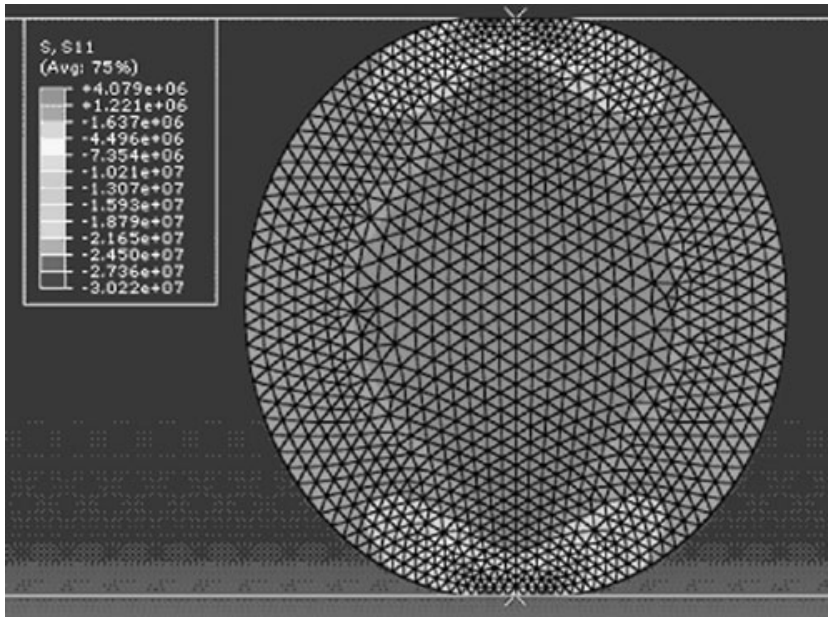

b)

Figura 4. Tensiones principales en configuraciones a (óvalo 0,9 ): a) $\theta=45^{\circ}$; b) $\theta=0^{\circ}$.

condiciones de contorno antes descriptas, el empleo de la simetría hizo necesaria la restricción de un grado de libertad (translación en el eje Z) en todos los nodos contenidos en el plano de referencia, representando los efectos de una mitad sobre la otra. Se consideró también la posición relativa del defecto respecto al eje de aplicación de la carga, descripta por el ángulo $\xi$ entre el plano que contiene al eje $Z$ y a los espesores máximo/mínimo, y el eje de carga.

El estudio de estos defectos permitió analizar la ubicación y dirección de las máximas tensiones normales generadas en la probeta y calcular el error en el valor de resistencia a la fractura acarreado por la presencia del defecto. El error relativo en la magnitud de dichas tensiones se calculó como la resta entre el valor obtenido analíticamente con la ecuación (1) para los valores de carga alcanzados en el modelo $(P)$, y las tensiones máximas obtenidas de las simulaciones.

\section{RESULTADOS Y DISCUSION}

En la Figura 4 se muestra la distribución de tensiones para dos valores del ángulo $\theta\left(0^{\circ}\right.$ y $\left.45^{\circ}\right)$ y una desviación de la circularidad de 0,9. La simulación de ensayos de compresión diametral sobre probetas ovaladas permitió demostrar que en todos los casos la tensión máxima en tracción se ubica en el centro de la probeta, como lo predice la teoría de Hertz, y que la dirección de la misma depende del ángulo $\theta$ (Figura 5a).

Se verificó que $\lambda$ aumenta con $\theta$ hasta alcanzar su máximo valor $\left(\lambda_{\text {máx }}\right)$ cuando $\theta=45^{\circ}$, independientemente del grado de ovalamiento. A partir de allí, un aumento de $\theta$ produce una disminución en el ángulo de inclinación de la tensión principal. Por su parte, el valor máximo del ángulo de inclinación de la tensión principal $\left(\lambda_{\max }\right)$ aumenta proporcionalmente al grado de ovalamiento. La dirección de la tensión principal máxima en el centro de la probeta se alinea con el eje $X$ para $\theta=0^{\circ}$ y $\theta=90^{\circ}$, configuraciones en las que se espera una fractura lineal que se inicie en el centro y propague hacia las zonas de carga formando superficies de fractura planas, al igual que en el modelo de Hertz. En el resto de los casos, se esperaría que la fractura iniciada en el centro del disco, propague en una dirección inclinada un ángulo $\lambda$ respecto al eje de aplicación de la carga. Para la máxima desviación de la circularidad que presentaron los discos estudiados (óvalo 0,96 ), el bajo valor de $\lambda$ obtenido (algo mayor a $2^{\circ}$ para el caso más desfavorable) hace difícil la constatación experimental de la inclinación del plano de la fractura (para el caso de las probetas que fracturaron diametralmente o por 'triple-cleft').

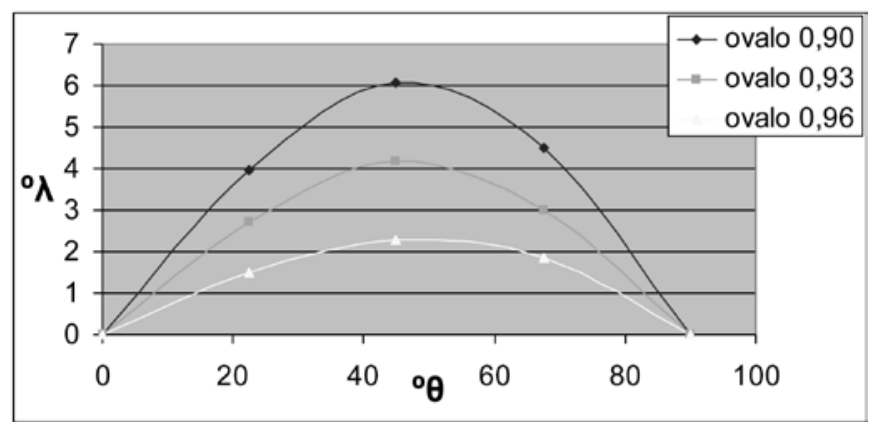

a)

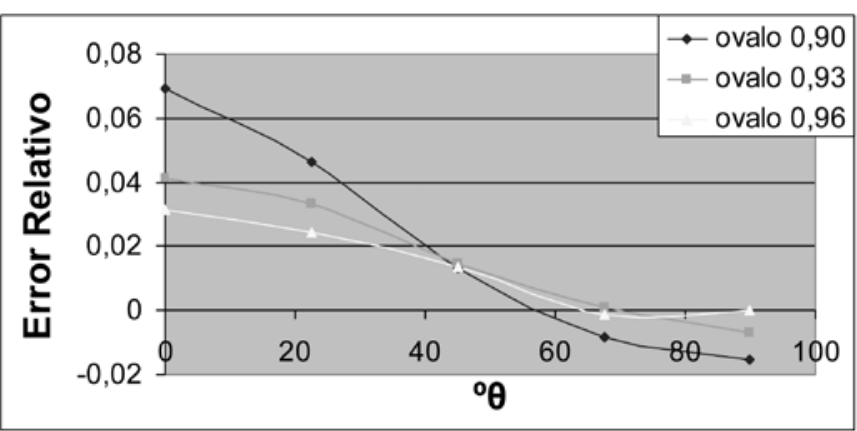

b)

Figura 5. Efectos de la desviación de la circularidad: a) variación de $\lambda$ en función de la posición de la probeta $(\theta)$; b) error relativo en las tensiones normales máximas. 
El cálculo de las tensiones máximas por la ecuación de Hertz considerando el diámetro medio del óvalo y el valor de carga registrado en cada simulación muestra discrepancias con los obtenidos numéricamente para las distintas posiciones del defecto (Figura 5b). Los valores de la tensión máxima se ven afectados por un error $\leq 4 \%$ si consideramos el máximo ovalamiento registrado en las probetas ensayadas $(0,96)$. El error es máximo para $\theta=0^{\circ}$ y tiende a disminuir a medida que este ángulo crece. Considerar una probeta ovalada como circular y calcular su resistencia con la ecuación de Hertz puede ocasionar las siguientes situaciones extremas, de acuerdo al ángulo de inclinación de los ejes del óvalo y de la carga evaluados: desde una subestimación $<2 \%$ hasta una sobrevaluación $<7 \%$.

Las tensiones de corte en el plano $X Y$ mostraron incrementos máximos menores al $8 \%$ para el grado de ovalamiento 0,9 respecto al caso libre de defecto, manteniéndose cercano a la zona de contacto entre plato y probeta y en dirección a $45^{\circ}$ respecto de la horizontal. Esto conduciría a aumentar la posibilidad de falla en esa región, lo cual es consistente con los patrones de fractura típicos exhibidos por estos discos, en los que frecuentemente se observa fractura en la zona de contacto.

Para el estudio del no-paralelismo de caras planas se consideraron los siguientes valores de $\beta$, teniendo en cuenta las desviaciones observadas en los discos ensayados: $1^{\circ} 47^{\prime} 24^{\prime \prime}$ $\left(1,79^{\circ}\right)$ y $0^{\circ} 53^{\prime} 42^{\prime \prime}\left(0,89^{\circ}\right)$. El valor $\xi=0^{\circ}$ corresponde a la situación en la que el máximo espesor de la probeta se halla en contacto con el plato inferior. Según el modelo numérico, la posición de la tensión principal máxima desarrollada en la probeta mantuvo su ubicación sobre el plano de la cara inclinada para todos los valores del ángulo $\xi$ considerados, como se muestra en la Figura 6 para el caso de $\beta=1,79^{\circ}$ y $\xi$ $=45^{\circ}$. Respecto al sistema de referencia adoptado, presentó desplazamientos en la dirección $X$ menores a $0,2 \mathrm{~mm}$, en tanto que los mayores desplazamientos se produjeron en la dirección Y (Figura 7a).

La ausencia de planoparalelismo $(\beta)$ afecta de manera similar a la posición de la tensión principal máxima, principalmente para $\xi>20^{\circ}$. Para la máxima desviación

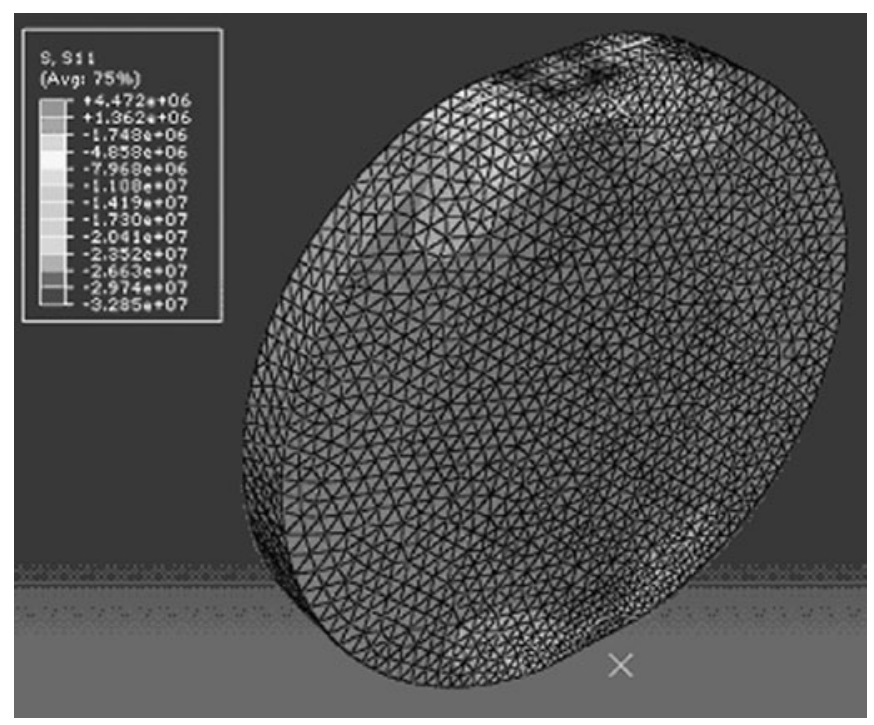

Figura 6. Distribución de tensiones $\sigma_{x x}$ para $\beta=1,79^{\circ}$ y $\xi=45^{\circ}$. simulada, esta tensión aparece a una distancia de $6 \mathrm{~mm}$ por debajo del centro del disco y se mantiene aproximadamente constante para el rango $0^{\circ}<\xi<60^{\circ}$. Para valores de $\xi>70^{\circ}$, la ubicación sufre un cambio abrupto, pasando a ubicarse a $6 \mathrm{~mm}$ del centro por encima del punto central (Figura 7a). No fue posible constatar este desplazamiento de la tensión máxima dado que no pudo determinarse la localización del defecto que originó la fractura.

Para el cálculo de la tensión principal máxima de tracción por la ecuación de Hertz se tomó como espesor del disco el valor medio, mientras el valor de la carga se extrajo de la solución del modelo. La comparación de las tensiones máximas calculadas y las arrojados por los modelos (Figura $7 b)$ pone en evidencia que el mayor error, que resulta en una subestimación cercana al $9 \%$, se produce para el mayor grado de desviación del paralelismo analizado, con desplazamientos angulares del eje de carga relativamente bajos $\left(\xi \sim 20^{\circ}\right)$. En este caso, el error resulta fuertemente dependiente de la orientación relativa del eje de carga $(\xi)$. La disminución de $\beta$ no garantiza menores errores en la estimación de la resistencia mecánica, a menos que el ángulo $\xi$ sea nulo.

Para el análisis de la desviación de la tensión de corte se tomó como referencia el valor de $\tau_{\text {máx }}$ obtenido por MEF para la condición de un disco con caras paralelas, considerando el valor de carga como se hizo anteriormente. La tensión de corte máxima asociada a fracturas en la región de contacto no se vio afectada en su posición, sin embargo sí se modifica su magnitud, produciendo un aumento $<10 \%$ cuando el ángulo de inclinación de cada superficie plana es de $1,79^{\circ}$, mientras que para $0,89^{\circ}$ el incremento fue del $2 \%$. Para los dos casos analizados, la ubicación de la tensión de corte máxima se registró siempre sobre la superficie de la cara

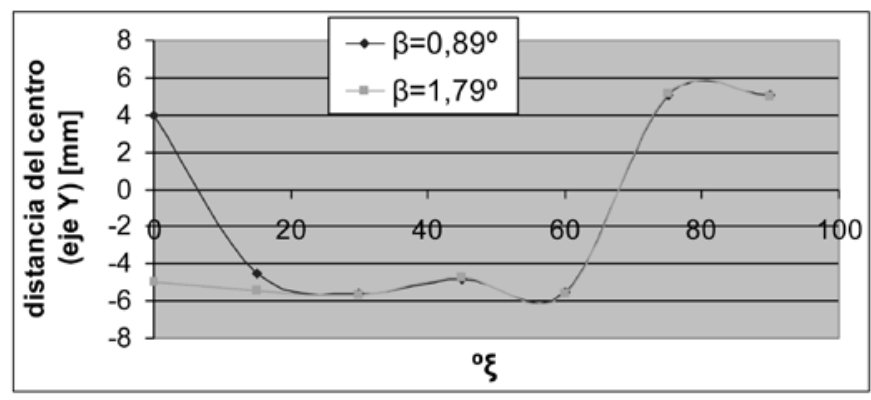

a)

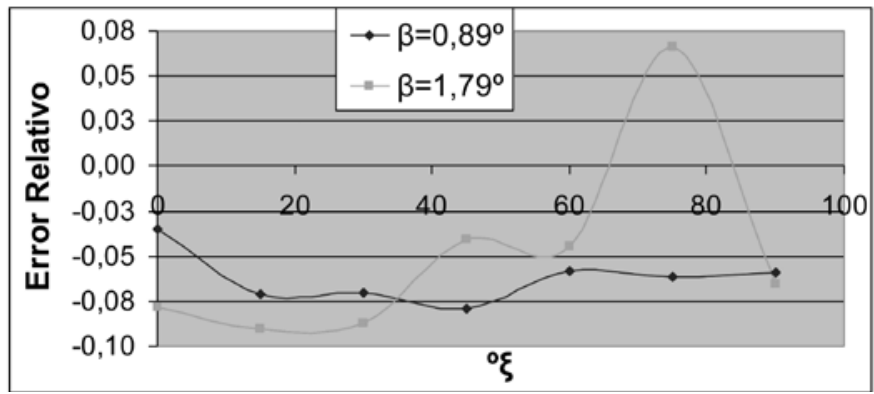

b)

Figura 7. Efecto del no-paralelismo de las caras planas del disco: a) variación en la ubicación de la tensión principal máxima en función del ángulo $\xi$; b) discrepancias entre el modelo de Hertz y el numérico. 
inclinada, donde hace contacto con el plato de compresión y forma con él un ángulo agudo. La influencia de la inclinación de las caras sobre la magnitud de las tensiones de corte aumentaría la susceptibilidad del material a fallar en la región de contacto, lo cual podría también explicar la alta frecuencia con la que ocurrió fractura en la zona de contacto por delaminación.

\section{CONLUSIONES}

El uso de una herramienta de cálculo como el método de elementos finitos posibilitó el análisis de condiciones diferentes a las supuestas en los modelos teóricos, detectadas durante el desarrollo de ensayos mecánicos en compresión diametral para la evaluación mecánica de discos porosos de cordierita. Si bien en una primera instancia se consideró un problema de elasticidad lineal, esta técnica permite introducir en futuras etapas modelos de comportamiento no-lineal, más cercano al que presenta este tipo de material, y el efecto de otros factores, no considerados en este primer modelo (ancho del área de contacto, por ejemplo).

En especial, se simularon desviaciones geométricas del disco como falta de circularidad y de paralelismo de las caras planas, que fueron las más frecuentes en la práctica. Los resultados obtenidos por cálculo permitieron evaluar posibles errores en la determinación de la resistencia mecánica empleando los modelos teóricos (Hertz) y aportar justificaciones adicionales a algunos de los resultados experimentales, en particular, en relación al tipo de fractura. Los errores resultaron en todos los casos menores al $10 \%$ para las desviaciones más extremas, con lo cual la aplicación de esta metodología se considera válida, en particular para los fines comparativos con los que fue utilizada en el estudio más amplio del cual forma parte este trabajo (8). La falta de planoparalelismo, que resulta la desviación geométrica que mayor error introduce en el cálculo de la resistencia mecánica, puede reducirse mediante procesos de mecanizado post-sinterizado, lo cual llevaría a mejorar la exactitud de los resultados.

\section{REFERENCIAS}

(1) A.R. Studart, U.T. Gonzenbach, E. Tervoort, L.J. Gauckler. Processing Routes to Macroporous Ceramics. A review. J. Am. Ceram. Soc., 89, 6, 1771-1789 (2006)

(2) X. Mao, S. Wang, S. Shimai. Porous ceramics with tri-modal pores preparated by foaming and starch consolidation. Ceram. Int., 34, 1, 107112 (2008)

(3) J.E. Bailey, N. A. Hill. The effect of porosity and microstructure on the mechanical properties of ceramics. Brit. Ceram. Soc., 15 (1970).

(4) R. H. Marion, J. K. Johnstone. A parametric study of the diametral compression test for ceramics. Am. Ceram. Bull., 56 (11), 998-1002 (1977).

(5) B.W. Darvel. Review uniaxial compression tests and the validity of indirect tensile strength. J. Mat. Sc., 25, 757-780 (1990).

(6) M.K. Fahad. Stresses and failure in the diametral compression test. J. Mat. Sc., 31, 3723-3729 (1996).

(7) N. Özkan, B.J. Briscoe. Characterization of die-pressed green compacts. J. Eur. Cer. Soc., 17, 697-711 (1997).

(8) L. J. Neergaard, D. A. Neergaard, M. S. Neergaard. Effective volume of specimens in diametral compression. J. Mat. Sc., 32, 2529-2533 (1997).

(9) A. T. Procopio, A. Zavaliangos, J.C. Cunningham. Analysis of the diametral compression test and the applicability to plastically deforming materials. J. Mat. Sc., 38, 3629-3639 (2003).

(10) C. Rocco, G.V. Guinea, J. Planas, M. Elices. Review of the splitting-test Standards from a fracture mechanics point of view. Cem. and Concr. Res., 21, 73-82 (2001).

(11) J. L. Amorós, V. Cantavella, J. C. Jarque, C. Felíu. Green strength testing of presses compacts: An analysis of the different methods. J. Eur. Cer. Soc., 28, 701-710 (2008).

(12) I. Iglesias, B. Acosta, R. Yu, G. Ruiz, M. Aineto, A. Acosta. Estudio de caracterización mecánica de probetas cerámicas a partir de una adaptación del ensayo brasileño. Materiales de Construcción, 61, 417-429 (2011).

(13) E. Saris, Z. Agioutantis, K. Kaklis, S. K. Kourkoulis. Numerical simulations of the cracked brazilian disc under diametral compression. Ed. Springer Berlin Heidelberg (2007).

(14) M. Pucheu. Evaluación mecánica de materiales cerámicos porosos mediante ensayos de compresión diametral. Proyecto Final, Ing. mecánica, Universidad Nacional de Mar del Plata, marzo 2009.

(15) M. L. Sandoval, M.A. Pucheu, M. H. Talou, A. G. Tomba M., M. A. Camerucci. Mechanical evaluation of cordierite precursor green bodies obtained by starch thermogelling. J. Eur. Cer. Soc., 29, 3307-3317 (2009).

(16) M. L. Sandoval, M. H. Talou, A. G. Tomba M., M. A. Camerucci. Mechanical testing of cordierite porous ceramics using high temperature diametral compression. J. Mat. Sc., 45, 14, 5109-5117 (2010).

(17) G. Rohr, M.A. Camerucci, A. N. Scian, A. L. Cavalieri. Respuesta mecánica a alta temperatura de materiales basados en cordierita. Actas CONAMET-SAM 2006, noviembre 2006, Santiago, Chile.

(18) M.A. Camerucci, G. Urretavizcaya, A.L. Cavalieri. Mechanical behavior of cordierite and cordierite-mullite materials evaluated by indentation techniques. J. Eur. Cer. Soc., 21, 1195-1204 (2001).

(19) R.H. Marion, J. K. Johnstone. A parametric study of the diametral compression test for ceramics. Am. Ceram. Bull., 56, 11, 998-1002 (1977).

(20) ASM Handbook Vol. 18. Friction lubrication and wear technology. ASM 1992.

Recibido: $21 / 9 / 2011$

Aceptado: 22/2/2012 


\section{Introducción a los esmaltes cerámicos}

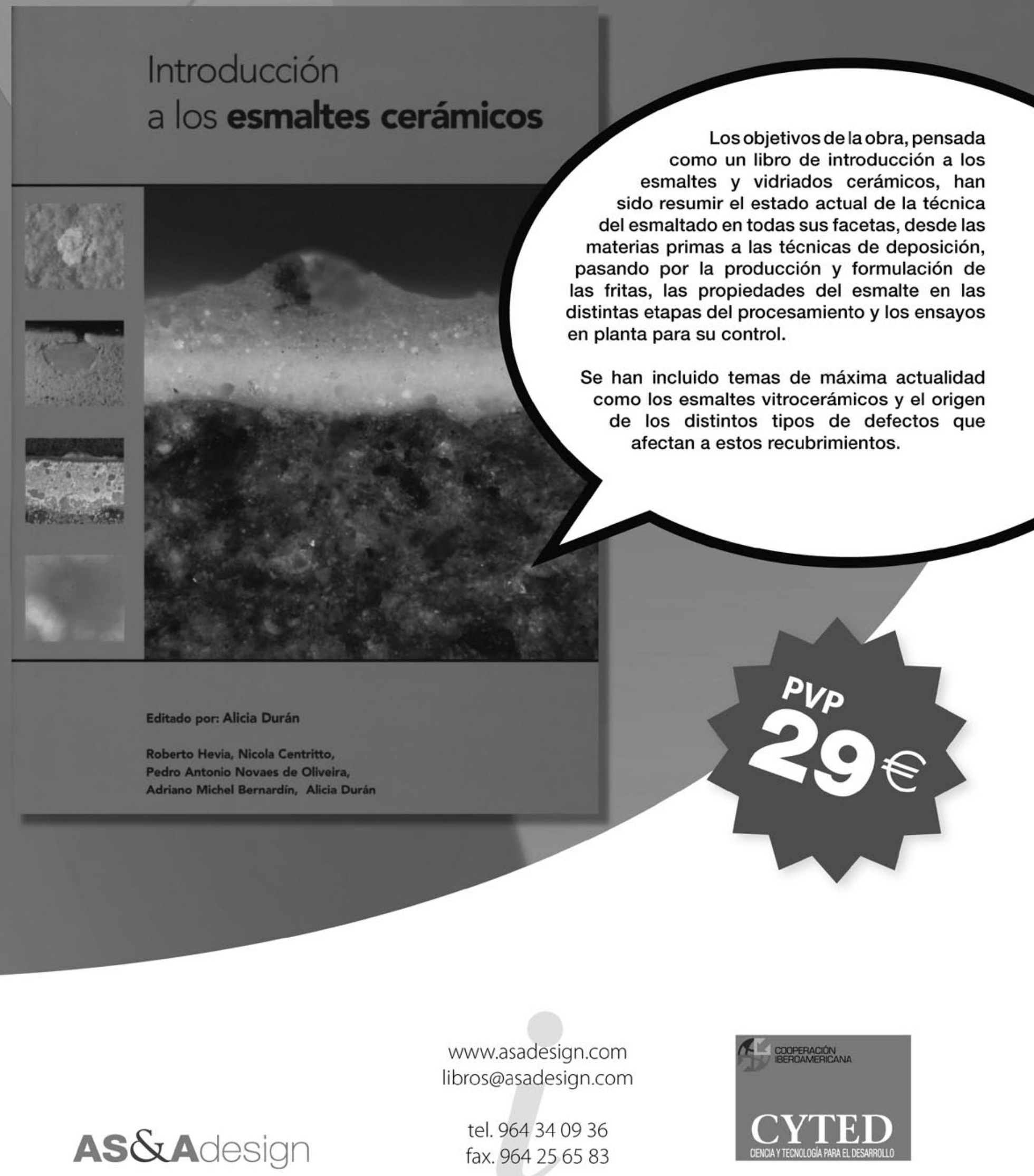

